Este trabajo ha obtenido un Accésit del Premio Estudios Financieros 2016 en la modalidad de Educación y Nuevas Tecnologías. El jurado ha estado compuesto por: don Antonio Bautista García-Vera, don Luis Alberto Guijarro Rojo, doña Ana Hidalgo Tena, Doña Blanca Lozano Cutanda y don Eugenio Martínez Falero. (Los trabajos se presentan con seudónimo y la selección se efectúa garantizando el anonimato de los autores)

\title{
EncyCloudPrado:
}

\section{posibilidades didácticas de las nuevas tecnologías para el acceso a la información en el ámbito museístico}

\section{Sumario}

1. Introducción

2. Estado de la cuestión

3. Material y métodos

4. Análisis y resultados

5. Conclusiones

6. Bibliografía

Anexo

\section{Extracto:}

El papel y el desarrollo de los museos en el mundo contemporáneo está estrechamente ligado a las posibilidades que ofrecen las tecnologías de la información y la comunicación (TIC). En línea con la vocación educadora de las instituciones museísticas, el presente trabajo ofrece un recurso didáctico para facilitar el acceso a la información y la visualización de fondos culturales en la era digital. En el marco de esta investigación se ha creado el prototipo EncyCloudPrado 2016, una instalación interactiva que permite realizar búsquedas en la versión online de la enciclopedia del Museo Nacional del Prado. Los resultados aparecen proyectados en una pantalla panorámica de 360 grados a modo de dinámicos collages de sonidos, documentos e imágenes, convirtiendo la navegación por la web en una experiencia espacial. El objetivo de este estudio consiste en analizar el grado de aceptación por parte de la generación $Z$ del uso de instalaciones interactivas en entornos inmersivos como posibles herramientas de aprendizaje. Asimismo, se valorará si este tipo de iniciativas supone un acicate para atraer a un mayor número de visitantes a los museos y favorecer el acceso a sus fondos.

Palabras clave: cultura participativa, educación informal, EncyCloudPrado, entorno inmersivo, innovación didáctica, museo, tecnologías de la información y la comunicación (TIC).

\footnotetext{
1 C. Fernández Castrillo, profesora titular en la Facultad de Ciencias Sociales y Humanidades de la Universidad a Distancia de Madrid (UDIMA).

2 B. Lintermann, profesor adjunto en la Facultad de Medios Creativos en la City University of Hong Kong.
}

El equipo investigador de este estudio agradece la colaboración prestada por Javier Pantoja Ferrari, jefe de área de Desarrollo Digital del Museo Nacional del Prado; la Fundación Amigos Museo del Prado; el ZKM (Center for Art and Media); y la profesora Almudena Rubio Cámara, coordinadora TIC del Instituto de Educación Secundaria (IES) Príncipe Felipe de Madrid. 


\section{EncyCloudPrado:}

\section{didactic possibilities of} new technologies

\section{for accessing information in}

\section{museums}

\section{Abstract:}

The role and the development of museums in the contemporary world is closely related to the possibilities offered by the information and communication technologies (ICT). In line with the educational vocation of museums as cultural institutions, this paper offers a didactic resource to provide an easier access to information and the visualization of the cultural background in the digital age. In the framework of this research it was created the prototype EncyCloudPrado (2016), an interactive installation that allows searches through the online Museo Nacional del Prado's encyclopedia. The results are projected on a 360-degree widescreen as dynamic collages of sounds, documents and images, turning web browsing into a spatial experience. The aim of this study is to determine the degree of acceptance of the use of interactive installations in immersive environments by the generation $\mathrm{Z}$ as possible learning tools. It will also be assessed if this type of initiatives may be an incentive to attract more visits to museums and to promote the access to their funds.

Keywords: participative culture, informal education, EncyCloudPrado, immersive environment, didactic innovation, museum, information and communication technologies (ICT).

[...] los museos [...] constituyen los contextos ideales para poner en marcha este tipo de experiencias de innovación docente debido a la mayor disponibilidad de recursos económicos, técnicos y humanos

\section{INTRODUCCIÓN}

En la actualidad, las posibilidades didácticas de las TIC en la relación del museo con el visitante abren un nuevo horizonte aún por explorar. En este trabajo se presenta una propuesta que se integra en el proceso de dinamización sociocultural que las instituciones museísticas están llevando a cabo. Su evolución de organismos depositarios de conocimiento a organizaciones para la instrucción y la divulgación cultural implica la adaptación a las necesidades de la sociedad contemporánea, todo un desafío desde el momento en que existe el compromiso no solo de exhibir el patrimonio, sino también de hacerlo comprensible a un público diverso (ICOM, 2007).

Se parte de la hipótesis de que los museos, en calidad de instituciones especializadas en educación informal, constituyen los contextos ideales para poner en marcha este tipo de experiencias de innovación docente debido a la mayor disponibilidad de recursos económicos, técnicos y humanos. Por ello, también se evaluará si este tipo de iniciativas contribuyen a fomentar las visitas a los museos y la consulta de los fondos disponibles.

Para llevar a cabo el proyecto, se ha procedido a la creación de EncyCloudPrado 2016, una instalación que permite realizar búsquedas de información en la enciclopedia online del Museo Nacional del Prado de forma innovadora. Mientras que la pantalla del ordenador únicamente permite acceder a los datos obtenidos desde una pequeña ventana y de forma lineal, EncyCloudPrado convierte la navegación por la web en una experiencia espacial, a la vez que brinda una atractiva visión del paisaje hipermedia en la red. Una vez concluida la etapa de producción y de montaje del prototipo, se ha procedido a la planificación del diseño de la investigación para la recogida de datos, partiendo de la selección de la muestra y de la elección de la técnica de obtención de la información para su posterior análisis estadístico. Por último, la propuesta de una actividad docente de carácter innovador dirigida a estudiantes de entre 15 y 19 años ha facilitado la recogida de datos, dando a conocer tanto su percepción inicial y conocimientos previos acerca de las instalaciones interactivas en entornos inmersivos como su valoración de EncyCloudPrado. 


\section{ESTADO DE LA CUESTIÓN}

Para llevar a cabo la investigación se ha procedido a realizar una revisión del marco teórico a partir de dos cuestiones fundamentales: el impacto de las TIC en el ámbito museístico y las estrategias para fomentar la participación del usuario en este contexto previstas en el Plan de Actuación 2013-2016 del Museo Nacional del Prado (Ministerio de Educación, Cultura y Deporte [MECD], 2015).

\subsection{El impacto de las TIC en el ámbito edu- cativo desde los museos}

Mediante este estudio se pretende averiguar en qué grado la posible desconexión existente entre las expectativas de los más jóvenes y unos recursos educativos no basados en la nueva gestión del conocimiento en la era digital puede constituir una seria amenaza para las instituciones museísticas de nuestro país.

Las investigaciones sobre las excursiones en grupo corroboran la necesidad de plantear alternativas a las visitas orientadas a un mero recorrido estructurado de forma lineal por las obras principales, para proporcionar un aprendizaje experiencial, personalizado y contextualizado (Vartiainen y Enkenberg, 2013; Tal y Morag, 2007). Por consiguiente, facilitar la inmersión en un determinado ambiente favorecerá la adquisición de conocimientos mediante la práctica del learning by doing (Lindquist y Long, 2011; Dede, 2005; Oblinger, 2003; Prensky, 2001).

Entre los principales exponentes de la creación de instalaciones interactivas con fines didácticos se encuentra Maria Roussou, fundadora del Departamento de Realidad Virtual de la Foundation of the Hellenic World (FHW) y defensora del inevitable impacto de este nuevo tipo de tecnología en la oferta educativa de los museos. Esta experta sostiene que las experiencias en espacios de navegación inmersivos resultan naturales para los más jóvenes debido a su mayor capacidad de abstracción y de adaptación a distintos espacios gráficos y conceptuales (Roussou, 2000).

Por tanto, las nuevas líneas de actuación para el fomento de la misión educativa desde los museos deben atender a las necesidades de la generación Z, comprendida por aquellos nacidos entre 1994 y 2010.

\section{Mediante este estudio se pretende averiguar en qué grado la posible desconexión existente entre las expectativas de los más jóvenes y unos recursos educativos no basados en la nueva gestión del conocimiento en la era digital puede constituir una seria amenaza para las instituciones museísticas de nuestro país}

\subsection{Plan de Actuación 2013-2016 del Mu- seo Nacional del Prado}

En el Plan de Actuación 2013-2016 del Museo Nacional del Prado, las líneas de acción previstas para dicho periodo persiguen fundamentalmente un aumento de los ingresos para compensar las limitaciones presupuestarias derivadas de la reducción de la aportación del Estado ${ }^{3}$ y el descenso del turismo. Entre las medidas de fomento para la promoción de las visitas, queda manifiesta la intención de "cumplir la vocación de servicio público y educativo del museo, ofreciendo la máxima accesibilidad y la mejor interpretación de la identidad e historia de las colecciones con el fin de facilitar la mejor experiencia a los diferentes tipos de públicos» (MECD, 2013, pág. 15). No obstante, la ampliación de la oferta educativa prevista se centra principalmente en el planteamiento de nuevos tours guiados y en la continuación de itinerarios a la colección permanente mediante la incorporación de temas transversales (MECD, 2013, págs. 45-47). El uso de las TIC para atraer al público juvenil y adolescente se presenta como un objetivo de carácter general, pero sin especifi-

\footnotetext{
${ }^{3}$ «• Los ingresos propios generados por el museo, como consecuencia del dinamismo de su actividad, han permitido pasar de los 15.064.000 euros obtenidos en 2007 a los 24.186 .000 euros que se van a alcanzar en 2012, lo que supone un incremento del 60,6\%.

- Por su parte, la aportación del MECD ha pasado de los 28.388.000 euros en 2007 a los 15.905 .000 euros en 2012, lo que supone una reducción del $44,0 \%$.

- El nivel de autofinanciación del organismo situado en el $34,7 \%$ en 2007 alcanzará, de acuerdo con el cierre provisional del ejercicio 2012, el 60,1\%» (MECD, 2013, pág. 7).
} 


\section{En esta investigación se pretende contribuir al objetivo estratégico de ufacilitar la visita al museo y la interpretación de sus colecciones para el público general, escolar y con necesidades especiales, poniendo a su disposición los instrumentos necesarios, con especial atención a las nuevas tecnologías» (MECD, 2013)}

carse medidas concretas al respecto. De hecho, hasta el momento, la utilización de las redes sociales o el desarrollo de aplicaciones se han enfocado principalmente a la difusión de la actividad diaria del museo.

Una de las acciones recientes más destacables ha sido el lanzamiento de un renovado website a comienzos del año 2016 que abarca toda la actividad educativa del museo con el fin de favorecer la interacción en red del usuario. Desde la sección «Aprende» se facilita el acceso a los siguientes apartados: «Escuela del Prado», con información acerca de actividades, becas y publicaciones; «Biblioteca, archivo y documentación», para la consulta del catálogo y de las normas de acceso y uso de los fondos; «Investigación», con actualizaciones sobre las restauraciones y los últimos estudios; y «Recursos», desde donde se proporciona acceso al boletín y a la enciclopedia online del Museo del Prado. La enciclopedia es un proyecto de la Fundación Amigos del Museo del Prado, fruto del trabajo de más de 130 especialistas internacionales, que reúne información sobre más de 1.600 artistas, 210 exposiciones y 166 obras maestras de la pinacoteca.

En esta investigación se pretende contribuir al objetivo estratégico de «facilitar la visita al museo y la interpretación de sus colecciones para el público general, escolar y con necesidades especiales, poniendo a su disposición los instrumentos necesarios, con especial atención a las nuevas tecnologías» (MECD, 2013, pág. 42). Para ello se ha creado la instalación interactiva EncyCloudPrado, que muestra de forma innovadora los contenidos de la enciclopedia online, una herramienta fundamental para el estudio y el conocimiento del museo.
Al examinar la aplicación de este tipo de medios al ámbito del aprendizaje se podrá comprobar si la interactividad en entornos inmersivos puede aportar un valor añadido al sector educativo, especialmente en el contexto museístico, al ser el que cumple con un mayor número de requisitos para poner en práctica actividades experimentales que precisan de una determinada infraestructura, junto a condiciones técnicas y de inversión económica.

\section{MATERIAL Y MÉTODOS}

La presente investigación se basa en un estudio de caso que se ha llevado a cabo en cuatro fases principales:

\section{A) Primera fase}

Diseño y producción del prototipo EncyCloudPrado, una instalación interactiva que permite proyectar en una pantalla panorámica de $360^{\circ}$ las búsquedas en la enciclopedia online del Museo Nacional del Prado.

\section{B) Segunda fase}

Al tratarse de un prototipo, y debido a las condiciones técnicas necesarias para su exhibición, ha sido necesario grabar un docuclip para mostrar el funcionamiento de la instalación. Esta pieza audiovisual ha requerido de un proceso de posproducción con trabajo de doblaje y grafismo electrónico incluido.

\section{C) Tercera fase}

A continuación, se ha llevado a cabo el diseño de la investigación adoptando un enfoque preexperimental a partir de una muestra no aleatoria, cuyos criterios de selección han sido:
[...] se ha creado la instalación interactiva EncyCloudPrado, que muestra de forma innovadora los contenidos de la enciclopedia online, una herramienta fundamental para el estudio y el conocimiento del museo 
- Perfil. Estudiantes de $3 .^{\circ}$ y $4 .^{\circ}$ de educación secundaria obligatoria (ESO) y de $1 .^{\circ}$ y $2 .^{\circ}$ de bachillerato con un nivel de conocimiento medio-alto en TIC. El tamaño de la muestra ha sido de 325 participantes.

- Lugar. Comunidad Autónoma de Madrid. Interesaba conocer el grado de acogida del prototipo en el territorio donde se ubica el Museo Nacional del Prado.

El IES Príncipe Felipe de Madrid ha sido seleccionado al haber obtenido el Premio Nacional de Educación 2015 a las Nuevas Tecnologías y por ser uno de los 15 institutos que forman parte del programa de innovación tecnológica de la Comunidad de Madrid desde el curso 2010-2011. Este centro educativo se ubica en el distrito Fuencarral-El Pardo, en el denominado barrio de La Paz. Su área de influencia se caracteriza por una fuerte heterogeneidad socioeconómica, englobando áreas netamente populares, como el barrio del Pilar, y otras con un perfil poblacional de clase media o media-alta, lo que otorga una mayor representatividad a la muestra.

Se ha procedido a la planificación de la recogida de datos mediante el diseño de un cuestionario online a través de Google Forms, que ha constado de 25 preguntas (abiertas y cerradas, de opción múltiple [véase anexo]) y que se ha estructurado del siguiente modo:

- Presentación. Breve explicación sobre los objetivos de la actividad e indicaciones básicas para su realización.

\section{El IES Príncipe Felipe de Madrid ha sido seleccionado al haber obtenido el Premio Nacional de Educación 2015 a las Nuevas Tecnologías y por ser uno de los 15 institutos que forman parte del programa de innovación tecnológica de la Comunidad de Madrid desde el curso 2010-2011}

\section{- Pretest:}

- Bloque I. Datos personales del usuario.

- Bloque II. Percepción inicial y conocimientos previos.

- Visionado docuclip. Tras las preguntas preliminares, desde el propio cuestionario se facilita el enlace al docuclip sobre EncyCloudPrado.

\section{- Postest:}

- Bloque III. Valoración de la instalación interactiva.

- Bloque IV. Sugerencias sobre futuras líneas de actuación.

\section{Cuadro 1. Fortalezas y debilidades de las encuestas por internet}

\begin{tabular}{|c|c|}
\hline \multicolumn{2}{|c|}{ Encuestas por internet } \\
\hline Fortalezas & Debilidades \\
\hline Bajo coste y mayor rapidez. & Dificultad en plantear preguntas abiertas. \\
\hline No se necesitan entrevistadores, evitando los posibles sesgos del entrevistador. & Mayor coste en la elaboración del cuestionario. \\
\hline Al entrevistado le produce mayor sensación de anonimato. & Riesgos derivados de posibles fallos informáticos. \\
\hline Permite acceder a las poblaciones de difícil acceso. & Necesidad de disponer de conexión a internet. \\
\hline Evita errores de codificación, saltos de preguntas, etc. & Es un medio poco motivador si no existe un reclamo o incentivo. \\
\hline \multirow[t]{2}{*}{ Facilita el análisis cuantitativo de la información recogida. } & Es muy impersonal. \\
\hline & Imposibilidad de encuestar a quienes no dispongan de esta tecnología. \\
\hline
\end{tabular}


Al adoptar la encuesta por internet como técnica de recogida de información, se ha intentado minimizar el impacto de las debilidades de este tipo de cuestionario (véase cuadro 1) mediante la integración de la actividad en el programa formativo en TIC del IES Príncipe Felipe de Madrid. La realización del ejercicio se ha llevado a cabo a lo largo de dos semanas lectivas y en todo momento se ha contado con la supervisión del profesorado, al que se le ha facilitado una serie de instrucciones para garantizar la uniformidad de criterios en su aplicación.

\section{D) Cuarta fase}

La presente investigación es de corte exploratoriodescriptivo, pues el objetivo principal consiste en describir un fenómeno dado, a partir de un estudio de encuesta, para proporcionar datos que constituyan una primera aproximación a la realidad observada. Recordemos que en este caso se ha valorado el grado de aceptación por parte de la generación $Z$ del uso de instalaciones interactivas en entornos inmersivos como posibles herramientas de aprendizaje para el acceso a los fondos museísticos y si este tipo de experiencias pueden atraer a un mayor número de visitantes.

\section{ANÁLISIS Y RESULTADOS}

Una vez identificados los objetivos de la investigación, se ha recabado documentación acerca de la política de actuación del Museo Nacional del Prado para evaluar la viabilidad de esta propuesta dentro de las actuales líneas de interés. Posteriormente, tras el diseño del prototipo EncyCloudPrado, se ha procedido a la recogida y al análisis de datos con el fin de dar sentido a la información obtenida para poder explicar el fenómeno objeto de estudio.

\subsection{Aprendizaje interactivo a través de ins- talaciones inmersivas}

A continuación, conoceremos en qué consiste EncyCloudPrado, un proyecto que pretende proporcionar una mayor visibilidad y facilidad de acceso a los recursos disponibles mediante una interfaz acorde a las necesidades cognitivas de los más jóvenes.

\subsection{EncyCloudPrado}

Junto a su uso potencial como herramienta educativa de carácter innovador, EncyCloudPrado también se sitúa a la vanguardia de la analítica visual y de la estética de la información, áreas de acción esenciales en un presente en el que urge gestionar un flujo de datos cada vez mayor (Fernández, 2012; Lintermann, 2012). El historial de las búsquedas realizadas en la enciclopedia online del Museo Nacional del Prado y los documentos e imágenes relacionados con los temas de interés se convierten en un paisaje envolvente mediante su proyección en una pantalla panorámica. A través de una sencilla interfaz, el usuario puede controlar la instalación, transformando así la navegación por la web en toda una experiencia física.

Un Apple iPod Touch está integrado en el mando táctil que ha sido diseñado especialmente para EncyCloudPrado, lo cual permite realizar nuevas búsquedas introduciendo las palabras clave mediante un teclado. Por ejemplo, al seleccionar el tríptico El jardín de las delicias (1500-1505) del artista Hieronymus Bosch, el Bosco, se muestra un texto con la biografía del pintor y documentación sobre sus obras, que copan el horizonte visual del público (véase figura 1).

A través de dos botones laterales se puede acceder a la información anterior, avanzar, y también es posible desplazarse por los documentos relacionados con el tema de interés que podrán ser proyectados en la pantalla panorámica (véase figura 2).

\section{[...] EncyCloudPrado también se sitúa a la vanguardia de la analítica visual y de la estética de la información, áreas de acción esenciales en un presente en el que urge gestionar un flujo de datos cada vez mayor [...] A través de una sencilla interfaz, el usuario puede controlar la instalación, transformando así la navegación por la web en toda una experiencia física}




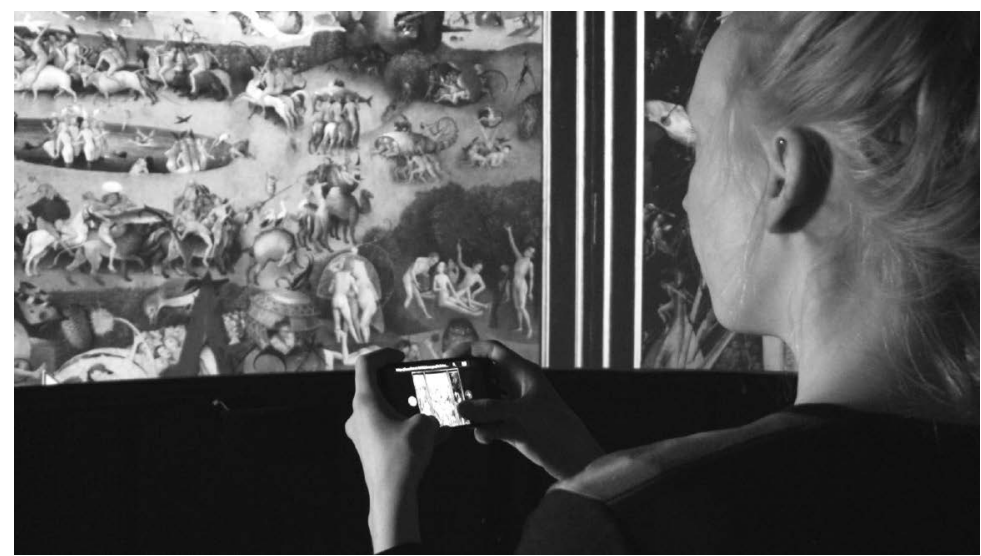

Fuente: elaboración propia.

\section{Figura 2. Mando táctil de EncyCloudPrado}

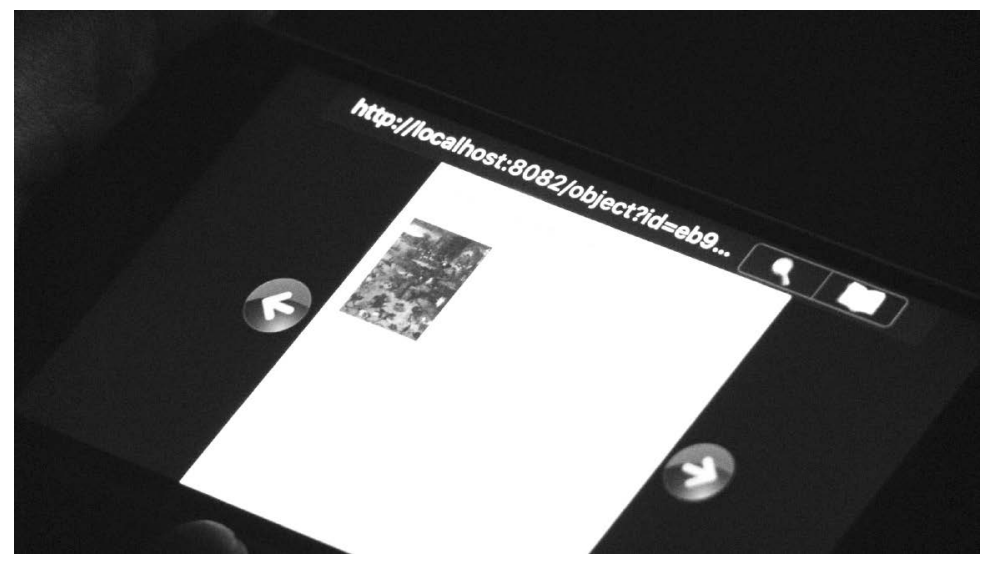

Fuente: elaboración propia. 


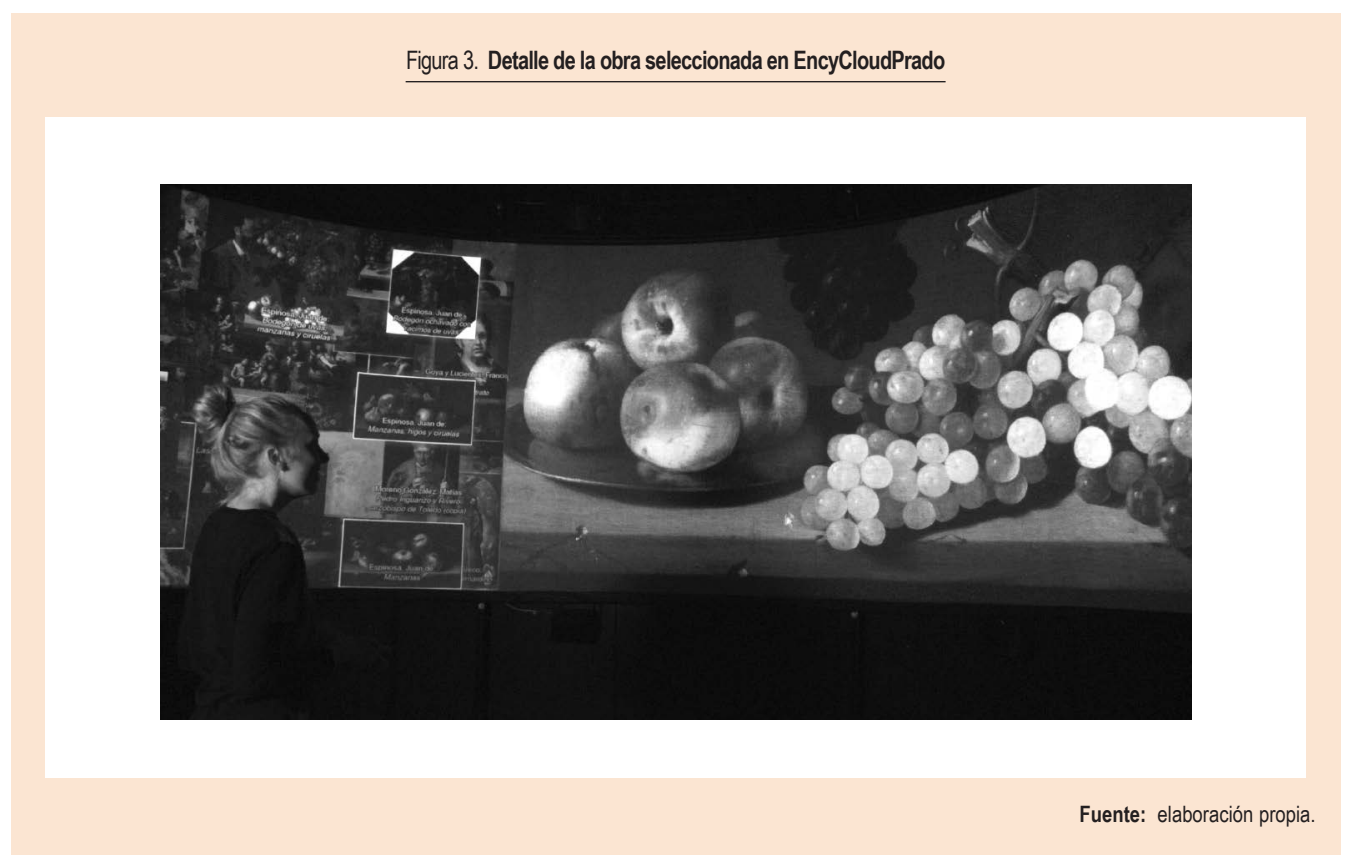

Incluso existe la posibilidad de ampliar la imagen en alta resolución para apreciar los detalles (véase figura 3). Gracias a un sensor, denominado Inertia Cube-Sensor, se detecta la orientación del usuario, de modo que este únicamente tendrá que mover el mando de arriba abajo y de izquierda a derecha para controlar el puntero. La información relacionada siempre aparecerá enfrente.

En EncyCloudPrado el usuario adoptará un papel protagonista al decidir los contenidos que aparecerán proyectados en ese momento, y que los futuros visitantes podrán contemplar al tiempo que realizan sus propias búsquedas. Al elegir una obra, el sistema sugiere también otras del mismo artista y de otros creadores que puedan resultarle interesantes. Así pues, los temas permanecen visibles y en la nube (cloud), de ahí el título. Las imágenes van acompañadas de sonido, y, al cambiar de tema, el ambiente musical varía. Por lo tanto, al entrar en la instalación el visitante podrá optar por revisar las búsquedas anteriores o ampliar el paisaje con nuevos temas, generando asi una galería hipermedia siempre nueva. De este modo, se pretende reflejar la cultura participativa y las dinámicas colaborativas presentes en las formas de acceso, selección y distribución de contenidos en la era digital.

\subsection{Análisis de los datos}

Una vez finalizado el diseño y el testeo del prototipo EncyCloudPrado, se ha procedido a realizar un estudio de encuesta. Esta modalidad de obtención de datos resulta especialmente útil para la predicción y la descripción de los nuevos fenómenos educativos. En este caso se pretendían valorar las reacciones de la muestra de población seleccionada por su interés como usuarios potenciales de la instalación.

\subsubsection{Datos personales del usuario}

El primer bloque del precuestionario se ha centrado en recopilar información para poder conocer el perfil de los 325 estudiantes encuestados del IES Príncipe Felipe. Los datos obtenidos del análisis estadístico revelan lo siguiente:

- El $57 \%$ son mujeres y el $43 \%$ hombres.

- La edad de los estudiantes participantes está comprendida entre los 15-21 años (véase figura 4).

- El $91 \%$ de los encuestados son de nacionalidad española y el $9 \%$ proviene de naciones pertenecientes a Europa, América Latina, África y Asia. 
- Según su nivel de estudios: el $15 \%$ cursa $3 .^{\circ}$ de ESO; el $14 \%, 4 .^{\circ}$ de ESO; el $59 \%, 1 .^{\circ}$ de bachillerato; y el $12 \%, 2 .^{\circ}$ de bachillerato (para consultar el desglose por especialidades véase figura 5 ).

- En cuanto a sus planes tras finalizar el ciclo de bachillerato: el $71 \%$ de los encuestados tiene previsto cursar estudios universitarios; el $13 \%$ aún no sabe qué hará; el $7 \%$, educación superior no universitaria; el $6 \%$, formación profesional; el $1 \%$ pretende incorporarse al mercado laboral; y el $2 \%$ restante contempla otras opciones.

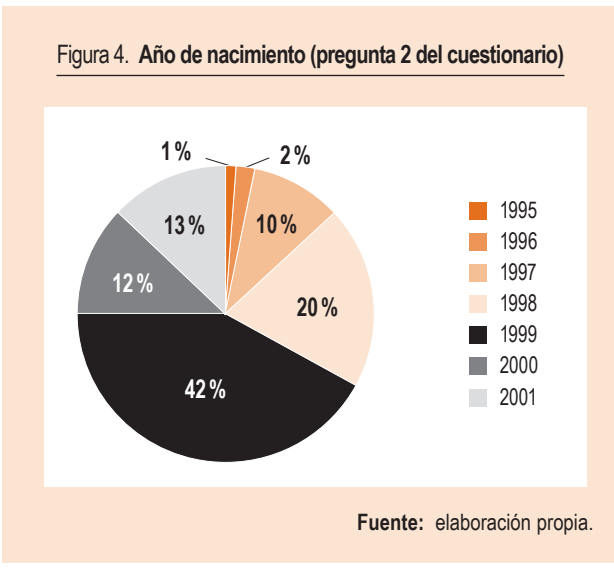

Figura 5. Nivel y especialidad académica actual (pregunta 4 del cuestionario)

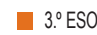

$4 .^{\circ} \mathrm{ESO}$

1. ${ }^{\circ}$ Bachillerato Artes

1..$^{\circ}$ Bachillerato Ciencias Sociales

- 1. ${ }^{\circ}$ Bachillerato Ciencia y Tecnología

2. ${ }^{\circ}$ Bachillerato Artes

2. ${ }^{\circ}$ Bachillerato Ciencias Sociales

$\square$ 2. ${ }^{\circ}$ Bachillerato Ciencia y Tecnología

Fuente: elaboración propia.

\subsubsection{Percepción inicial y conocimientos pre- vios de los encuestados}

Con anterioridad al visionado del docuclip, se ha estimado oportuno conocer las apreciaciones iniciales de los encuestados acerca de la presencia de las TIC en los museos y sus preferencias en el uso de los nuevos medios. De cara a la posible implementación de EncyCloudPrado, se han obtenido datos significativos en relación con la frecuencia de visitas a los museos y la realización de actividades educativas en estas instituciones. El análisis estadístico arroja los siguientes resultados:

- El $8 \%$ nunca ha ido al Museo del Prado; el $6 \%$ ha ido el último mes; el $18 \%$, hace menos de 3 meses; el $6 \%$, hace menos de 6 meses; el $24 \%$ ha acudido en el último año; el $15 \%$, hace 2 años, y el $23 \%$ hace más de 3 años (véase figura 6 ).

- En cuanto a la participación en actividades educativas organizadas por museos durante los últimos tres años, únicamente un $30 \%$ recuerda que estuviera presente el uso de las nuevas tecnologías.

- En relación a las páginas web de los museos, tan solo el $1 \%$ afirma consultarlas con regularidad; el $51 \%$ nunca las ha visitado; el $26 \%$, poco; y el $22 \%$, algo 0 bastante (véase figura 7 ).

\section{Figura 6. Frecuencia de visita al Museo Nacional del Prado (pregunta 6 del cuestionario)}

Nunca he ido

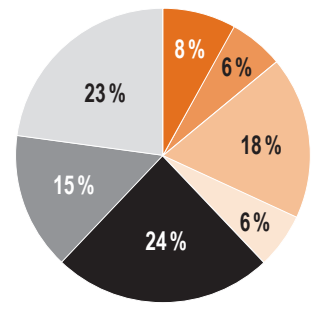

En el último mes

- Hace menos de 3 meses

Hace menos de 6 meses

- Hace menos de 1 año

Hace 2 años

Hace más de 3 años 
Figura 7. Frecuencia de consulta de páginas web de museos (pregunta 12 del cuestionario)

Nunca/Casi nunca

Poco

Algo

Bastante

Muy a menudo

Fuente: elaboración propia.

- La parte final del precuestionario incluía dos preguntas abiertas para conocer el grado de familiarización de los estudiantes con los conceptos «instalación interactiva» y «entorno inmersivo». En el primer caso, el 10\% manifestó no conocer su significado y aquellos que contestaron se centraron principalmente en destacar un alto grado de partici- pación, dinamismo y su finalidad lúdica. El $32 \%$ no supo responder a la segunda pregunta y quienes lo hicieron asociaron los entornos inmersivos con la realidad virtual.

\subsubsection{Valoración de la instalación interactiva según los encuestados}

Tras el visionado del docuclip, a través del poscuestionario se ha pretendido averiguar la opinión de los encuestados acerca de EncyCloudPrado y el grado de mejora de sus conocimientos sobre las instalaciones interactivas y los entornos inmersivos. Los resultados obtenidos han sido los siguientes:

- El grado de satisfacción general con EncyCloudPrado ha sido elevado: el $70 \%$ de los participantes estarían entre bastante y muy interesados en poder disfrutar de esta instalación al acudir al Museo Nacional del Prado; en un $70 \%$ de los casos consideran que es de bastante 0 de muy fácil manejo; la opción de poder seleccionar los contenidos proyectados está valorada entre bastante y muy positivamente en un $74 \%$; el acceso a un espacio inmersivo se aprecia entre mucho y bastante en un $63 \%$ frente a las posibilidades ofrecidas por el 3D $(60 \%)$ o las instalaciones interactivas $(57 \%)$ (véanse figuras 8.1 y 8.2 ).

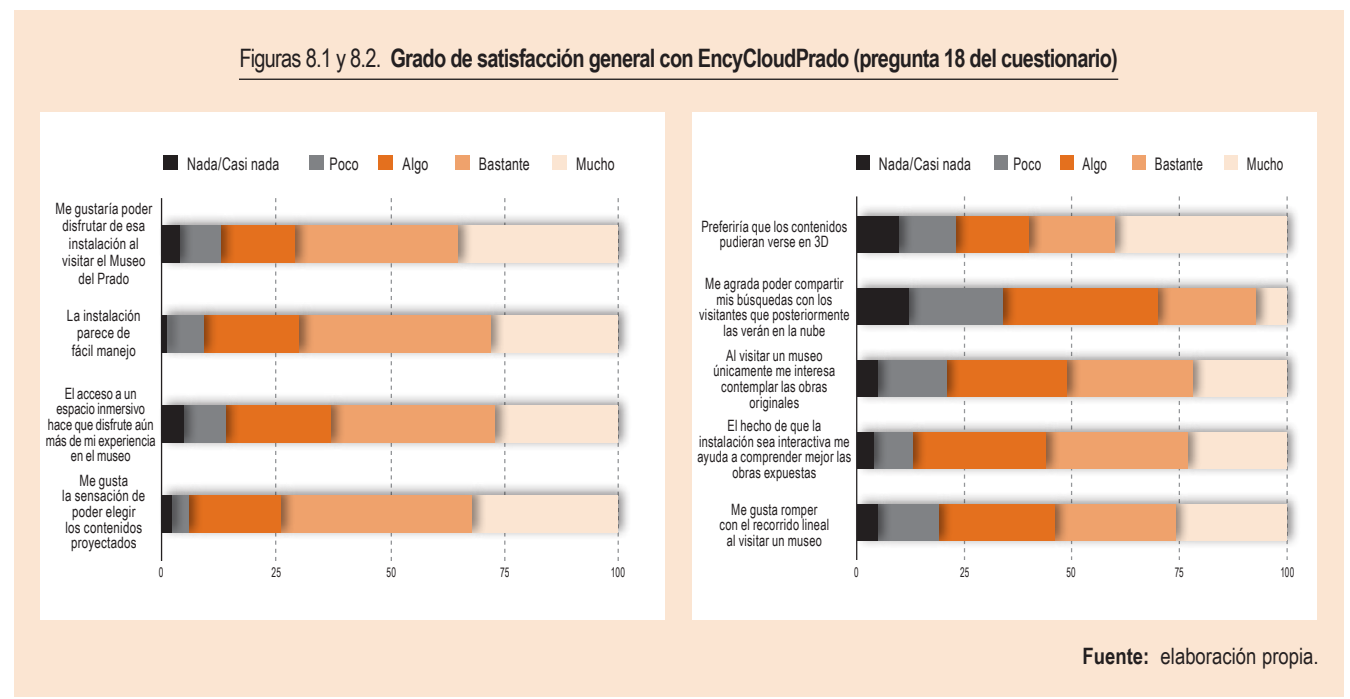


- La mejora en la capacidad para explicar en qué consiste una instalación interactiva tras el visionado del docuclip queda reflejada en que el $32 \%$ de los encuestados considera que podría definir el concepto con bastante precisión; el $38 \%$, con precisión; el $6 \%$, con mucha precisión; el $20 \%$ piensa que podría ser algo preciso; y el $4 \%$, nada preciso. En lo relativo a los entornos inmersivos, se registra un avance aún mayor, pues el $37 \%$ podría definir con bastante precisión en qué consisten; el $34 \%$, con precisión; el $8 \%$, con mucha precisión; el $19 \%$, con algo de precisión; y únicamente el $2 \%$, con ninguna precisión.

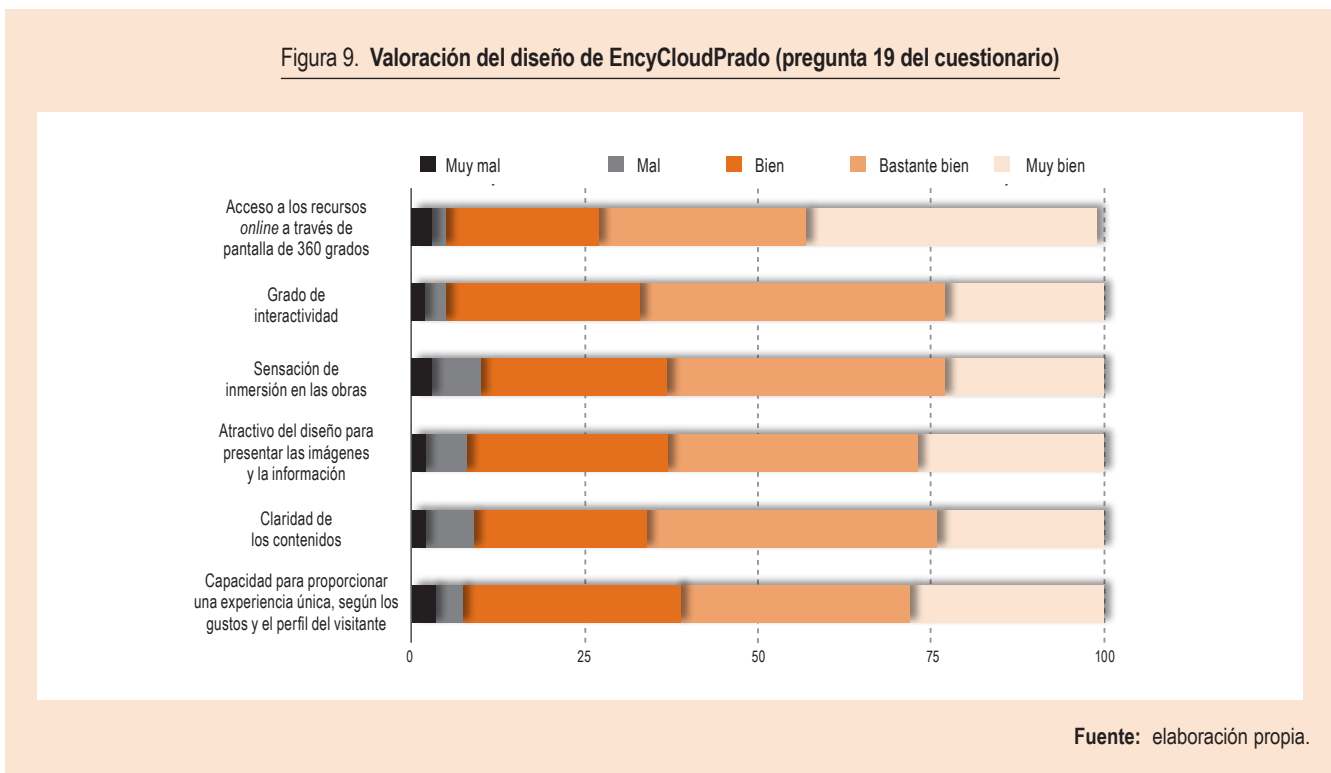

Figura 10. Grado de conformidad con los siguientes aspectos relacionados con el acceso a la información en EncyCloudPrado (pregunta 20 del cuestionario)

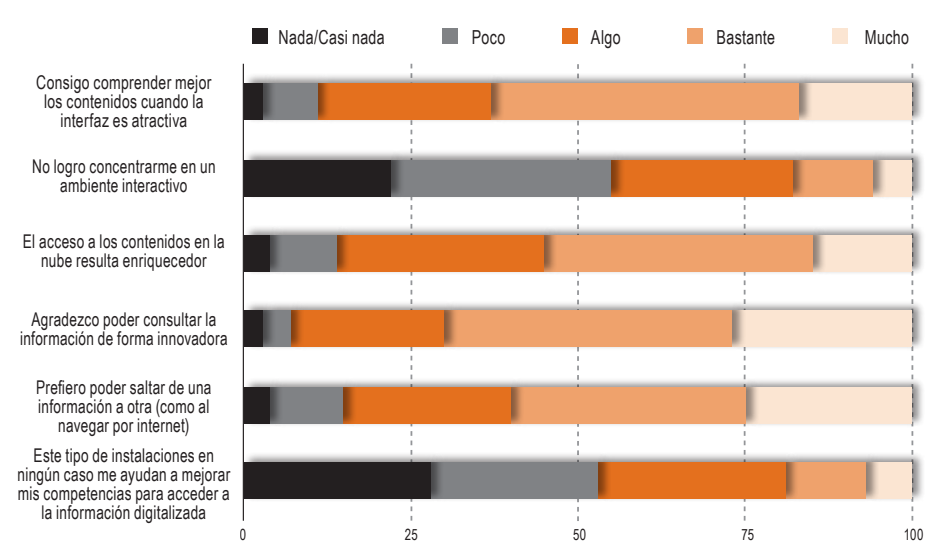




\subsubsection{Sugerencias sobre futuras líneas de actuación}

Por último, resultaba importante comprender en qué modo se podría mejorar el planteamiento y presentación de EncyCloudPrado de cara a su posible integración en la sede del Museo Nacional del Prado. A partir del análisis estadístico, estos son los datos que hemos obtenido:

- En cuanto al punto del recorrido en el que los encuestados preferirían acceder a la instalación, el $32 \%$ ha elegido que desearían acceder a la mitad del recorrido; el $30 \%$ seleccionó al final de la expo-

\section{CONCLUSIONES}

Entre los resultados más relevantes recabados a partir del análisis estadístico-descriptivo, en primer lugar, cabe destacar la necesidad de seguir promoviendo el desarrollo de actividades didácticas que puedan resultar atractivas para el $23 \%$ de los jóvenes encuestados que aún no conocen los servicios educativos ofertados por los museos de nuestro país.

Para evitar la anteriormente mencionada desconexión entre las expectativas de este sector de la muestra y unos recursos no basados en la nueva gestión del conocimiento en la era digital, urge promover el uso de las TIC en este ámbito, pues, según los encuestados, actualmente tiene una baja presencia (PQ8). Una realidad que, sin embargo, se pretende mantener tal y como hemos podido comprobar en los planes de actuación previstos por instituciones tan influyentes como el Museo Nacional del Prado (MECD, 2013, págs. 45-47). No obstante, como ya se ha comentado, este centro sí está llevando a cabo una importante labor de renovación de su website para la mejora de la interacción e implicación del usuario mediante la incorporación a su base de datos de más de 15.000 obras con su correspondiente documentación y la progresiva apertura de estos datos para su reutilización por terceros (open data) (MECD, 2013, págs. 49-50). Esta medida resulta de especial relevancia si se tiene en cuenta que el $51 \%$ de los participantes nunca han accedido a las páginas web de los museos (véase figura 7).

Los datos obtenidos revelan que las medidas de mejora para la incorporación de las TIC que se plantean actualmente desde el Museo Nacional del Prado (MECD, 2013, pág. 51), basadas en el podcasting y en sición; el $27 \%$, al inicio de la visita; y se dan otra serie de posibilidades en un $11 \%$.

- En lo referente al diseño, el $72 \%$ está entre bastante y muy satisfecho con el acceso a los recursos online a través de una pantalla de 360 grados; el grado de interactividad $(67 \%)$ y la claridad de los contenidos (65\%) son otras de las categorías mejor valoradas; mientras que el poder compartir las búsquedas con el resto de visitantes no es una opción que resulte prioritaria, pues el $34 \%$ le otorga poco o ningún valor (para una mayor profundización, véase figura 10).

el streaming de contenidos y actividades educativas 0 en la oferta de juegos a través de redes sociales, no resultan de tanto interés como la creación de espacios inmersivos de realidad virtual, aumentada o multisensorial. Por ello, EncyCloudPrado ofrece una serie de posibilidades que hay que tener en cuenta como recurso didáctico al valorarse positivamente por los encuestados el uso de una herramienta interactiva que ayude a comprender mejor las obras expuestas y genere una sensación de inmersión en los contenidos de interés que pueden llegar a resultar más atractivos desde una interfaz de este tipo (véanse figuras 8.2 y 9). Por otro lado, cabe destacar que los estudiantes hayan mani-
Los datos obtenidos revelan que las medidas de mejora para la incorporación de las TIC que se plantean actualmente desde el Museo Nacional del Prado [...], basadas en el podcasting y en el streaming de contenidos y actividades educativas o en la oferta de juegos a través de redes sociales, no resultan de tanto interés como la creación de espacios inmersivos de realidad virtual, aumentada o multisensorial 
festado que estas instalaciones ayudan a mejorar significativamente sus competencias para acceder a la información digitalizada al poder saltar de un contenido a otro, siguiendo un modus operandi análogo al de la

\section{BIBLIOGRAFÍA}

Dede, C. [2005]: «Planning for neomillennial learning styles», Educause Quarterly, 28 (1), págs. 7-12.

Fernández Castrillo, C. [2012]: «Jeffrey Shaw, un pionero en el arte de los nuevos medios», en P. Amador, M. ${ }^{a}$ R. Ruiz, T. López y J. Cubas (eds.), Imagen, cultura y tecnología: medios, usos y redes. Actas del Segundo Congreso Internacional sobre Imagen, Cultura y Tecnología, Madrid: Instituto de Cultura y Tecnología, UC3M, págs. 24-33.

ICOM [2007]: «Definición del museo». Disponible en: http://icom.museum/la-vision/definicion-del-museo/ L/1/ [Consultado: marzo de 2016].

Lindquist, T. y Long, H. [2011]: «How can educational technology facilitate student engagement with online primary sources? A user needs assessment», Library Hi Tech, 29 (2), págs. 224-241. DOI 10.1108/0737 8831111138152

Lintermann, B. [2012]: «Beyond Cinema», Proceedings of International Symposium on Diversifying Digital Film and New Media, Corea: Hongik University.

MECD [2013]: Plan de actuación 2013-2016. Museo Nacional del Prado. Disponible en: https://www.museodelprado.es/museo/plan-de-actuacion [Consultado: 14 de marzo de 2017]. navegación por internet (véase figura 10). Estos resultados corroboran la necesidad de adaptar las líneas de actuación en materia de actividades formativas a los nuevos procesos cognitivos de la generación $Z$.

[2015]: Anuario de estadísticas culturales 2015, Secretaría General Técnica: Subdirección General de Documentación y Publicaciones.

Oblinger, D. [2003]: «Boomers, gen-xers, and millennials: understanding the new students», Educause Review, 37 (4), págs. 37-47.

Prensky, M. [2001]: «Digital natives, digital immigrants», On the Horizon, 9 (5), págs. 1-6.

Roussou, M. [2000]: «Immersive interactive virtual reality and informal education», Proceedings of user interfaces for all: interactive learning environments for children, Atenas.

Tal, T. y Morag, O. [2007]: «School visits to natural history museums: teaching or enriching?», Journal of Research in Science Education, 44 (5), págs. 747-769.

Torrado Fonseca, M. [2009]: «Estudios de encuesta», en R. Bisquerra Alzina (coord.), Metodología de la investigación educativa, Madrid: La Muralla, págs. 231-257.

Vartiainen, H. y Enkenberg, J. [2013]: «Learning from and with museum objects: design perspectives, environment, and emerging learning systems», Education, Technology, Research and Development, 61 (5), págs. 841-862. DOI 10.1007/s11423-013-9311-8. 


\section{ANEXO}

\section{BLOQUE I. DATOS PERSONALES DEL USUARIO}

1. Sexo
$\square$ Mujer
Hombre

2. Año de nacimiento
1995
1996
1997
$\square 1998$
1999
$\square 2000$
2001

\section{Nacionalidad}

\section{Española}

$\square$ Otra

\section{Nivel y especialidad académica}

\begin{tabular}{|c|c|c|c|c|}
\hline $\begin{array}{l}1 .^{0} \text { de bachille- } \\
\text { rato en Artes }\end{array}$ & $\begin{array}{l}\square 1 .^{\circ} \text { de bachille- } \\
\text { rato en Cien- } \\
\text { cias Sociales }\end{array}$ & $\begin{array}{l}\square 1 .^{\circ} \text { de bachille- } \\
\text { rato en Ciencias } \\
\text { y Tecnología }\end{array}$ & $\begin{array}{l}\square 2 .^{\circ} \text { de bachille- } \\
\text { rato en Artes }\end{array}$ & $\begin{array}{l}\square 2 .^{\circ} \text { de bachille- } \\
\quad \text { rato en Ciencias } \\
\quad \text { Sociales }\end{array}$ \\
\hline
\end{tabular}

5. Planes tras finalizar los estudios de bachillerato

\begin{tabular}{|c|c|c|c|c|c|}
\hline $\begin{array}{l}\text { Incorporación } \\
\text { al mundo del } \\
\text { trabajo }\end{array}$ & $\begin{array}{l}\square \text { Formación } \\
\text { profesional }\end{array}$ & $\begin{array}{l}\square \text { Educación } \\
\text { superior no } \\
\text { universitaria }\end{array}$ & $\begin{array}{l}\square \text { Estudios uni } \\
\text { versitarios }\end{array}$ & $\begin{array}{l}\square \text { No sabe/no } \\
\text { contesta }\end{array}$ & $\begin{array}{c}\square \text { Otros planes } \\
\text { (especificar) }\end{array}$ \\
\hline
\end{tabular}

\section{BLOQUE II. PERCEPCIÓN INICIAL Y CONOCIMIENTOS PREVIOS}

\section{6. ¿Cuándo fue la última vez que visitaste el Museo del Prado?}

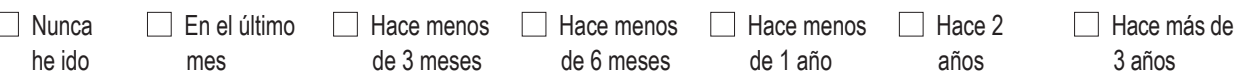

7. ¿En cuántas actividades educativas organizadas por museos has participado en los últimos 3 años?
$\square$ Ninguna
De 1 a 3
De 4 a 6
De 7 a 9
Más de 10

\section{En caso afirmativo, ¿estaba presente el uso de las nuevas tecnologías?}
Sí
$\square$ No
No lo recuerdo

9. Indica el grado de frecuencia con el que utilizas los siguientes medios (1 [Nunca/Casi nunca] y 5 [Muy a menudo])

$$
\begin{aligned}
& \text { 1. Nuncal } \\
& \text { Casinunca }
\end{aligned}
$$

Libros (incluidos e-books)

Revistas especializadas 


\section{BLOQUE II. PERCEPCIÓN INICIAL Y CONOCIMIENTOS PREVIOS}

.......

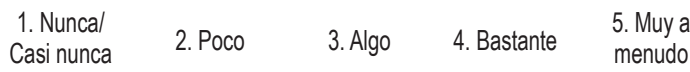

Películas

Televisión tradicional

Radio

Prensa online

Televisión a la carta

Podcasts

Páginas web de instituciones culturales

Redes sociales

YouTube

Blogs

Wikis

Aplicaciones de realidad aumentada/virtual

Instalaciones interactivas/inmersivas

Videojuegos (serious games)

10. En el contexto educativo, ¿en qué medida consideras que los siguientes medios audiovisuales pueden contribuir a que el proceso de aprendizaje te resulte más atractivo y, al mismo tiempo, puedas alcanzar los objetivos docentes? (1 [Nada/Casi nada] y 5 [Mucho])

\begin{tabular}{|c|c|c|c|}
\hline 1. Nada/ & 2. Poco & 3. Algo & 4. Bastante \\
\hline
\end{tabular}

Películas

Televisión tradicional

Radio tradicional

Prensa online (hipermedia)

Televisión a la carta

Podcasts

Páginas web de instituciones culturales

Redes sociales

YouTube

Blogs (hipermedia)

Wikis (hipermedia)

Aplicaciones de realidad aumentada/virtual

Instalaciones interactivas/inmersivas

Videojuegos (serious games)

$\begin{array}{ll}\square & \square \\ \square & \square \\ \square & \square \\ \square & \square \\ \square & \square \\ \square & \square \\ \square & \square \\ \square & \square \\ \square & \square \\ \square & \square \\ \square & \square \\ \square & \square \\ \square & \square \\ \square & \square\end{array}$




\section{BLOQUE II. PERCEPCIÓN INICIAL Y CONOCIMIENTOS PREVIOS}

......

11. Según la Organización de las Naciones Unidas para la Educación, la Ciencia y la Cultura (UNESCO), los recursos educativos abiertos (REA) están dirigidos a la enseñanza y al aprendizaje (materiales de estudio, manuales, streaming de vídeos, aplicaciones multimedia, podcasts, etc.) y están plenamente disponibles de forma gratuita a través de internet. A partir de esta definición, responde a las siguientes cuestiones (1 [Nada/ Casi nada] y 5 [Mucho])

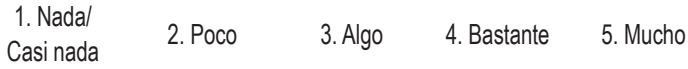

¿Te ha quedado clara la definición de los REA?

¿Ya conocías la existencia de los REA?

¿Has empleado alguna vez los REA?

¿Sabes cómo localizar los REA?

¿En qué medida resulta importante el diseño de la

interfaz para acceder al contenido de los REA?

¿Crees que te resultarán útiles los REA en el futuro?

12. ¿Con qué frecuencia sueles consultar las páginas web de museos?
Nunca/Casi nunca
Poco
Algo
Bastante
Muy a menudo

13. Define brevemente qué es una «instalación interactiva»

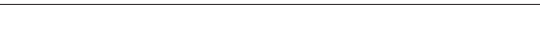

14. Explica con tus propias palabras qué es un «entorno inmersivo»

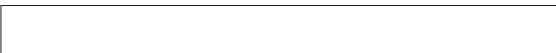

15. Expón cuál es tu nivel de satisfacción en relación a la presencia actual de las nuevas tecnologías en los museos
Ninguno
Poco
Algo
Bastante
Mucho

16. ¿En qué grado crees que los siguientes usos de las tecnologías harían más atractiva tu visita a un museo? (1 [Nada/Casi nada] y 5 [Mucho])

$\begin{array}{llll}\text { 1. Nadal } & \text { 2. Poco } & \text { 3. Algo } & \text { 4. Bastante } \\ \text { Casi nada } & \text { 5ucho }\end{array}$

Aplicación de realidad aumentada

Aplicación a modo de guía del museo

Instalación interactiva

Espacio de realidad virtual 


\section{BLOQUE II. PERCEPCIÓN INICIAL Y CONOCIMIENTOS PREVIOS}

$\ldots / \ldots$

\begin{tabular}{|c|c|c|c|}
\hline $\begin{array}{l}\text { 1. Nadal } \\
\text { Casi nada }\end{array}$ & 2. Poco & 3. Algo & 4. Bastante \\
\hline
\end{tabular}

Proyección inmersiva multisensorial

Juegos a través de redes sociales

Aplicación para ampliar información

Acceso desde tu móvil a streaming y podcasting de contenidos extra

Si lo deseas, indica cualquier otra tecnología que consideres especialmente relevante y que no esté incluida en el listado anterior (especificar)

\section{BLOQUE III. VALORACIÓN DE LA INSTALACIÓN INTERACTIVA}

\section{7. ¿Qué es lo que más te ha llamado la atención de EncyCloudPrado?}

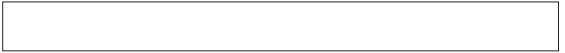

18. Indica en qué grado estás de acuerdo con las siguientes afirmaciones en relación con la instalación EncyCloudPrado (1 [Nada/Casi nada] y 5 [Mucho])

\begin{tabular}{|c|c|c|c|}
\hline 1. Nada/ & 2. Poco & 3. Algo & 4. Bastante \\
\hline
\end{tabular}

Me gustaría poder disfrutar de esta instalación al visitar el Museo del Prado

La instalación parece de fácil manejo

El acceso a un espacio inmersivo hace que disfrute aún más de mi experiencia en el museo

Me gusta la sensación de poder elegir los contenidos proyectados

Preferiría que los contenidos pudieran verse en 3D Me agrada poder compartir mis búsquedas con los visitantes que posteriormente las verán en la nube

Al visitar un museo únicamente me interesa contemplar las obras originales

El hecho de que la instalación sea interactiva me ayuda a comprender mejor las obras expuestas Me gusta romper con el recorrido lineal al visitar un museo

D

$$
\begin{aligned}
& \square \\
& \square \\
& \square \\
& \square \\
& \square \\
& \square \\
& \square \\
& \square
\end{aligned}
$$$$
\text { ....... }
$$ 


\section{BLOQUE III. VALORACIÓN DE LA INSTALACIÓN INTERACTIVA}

$\ldots / \ldots$

19. Valora los siguientes aspectos relacionados con el diseño de la instalación EncyCloudPrado (1 [Muy mal] y 5 [Muy bien])
1. Muy mal
2. Mal
3. Bien
4. Bastante bien
5. Muy bien

Acceso a los REA a través de una pantalla de 360 grados

Grado de interactividad

Sensación de inmersión en las obras

Atractivo del diseño para presentar las imágenes y

la información

Claridad de los contenidos

Capacidad para proporcionar una experiencia úni-

ca, según los gustos y el perfil del visitante

20. Indica en qué grado estás de acuerdo con los siguientes aspectos relacionados con el acceso a la información a través de la instalación EncyCloudPrado (1 [Nada/Casi nada] y 5 [Mucho])

$\begin{array}{llll}\begin{array}{l}\text { 1. Nadal } \\ \text { Casi nada }\end{array} \text { 2. Poco } & \text { 3. Algo } & \text { 4. Bastante } & \text { 5. Mucho }\end{array}$

Consigo comprender mejor los contenidos cuando la interfaz es atractiva

No logro concentrarme en un ambiente interactivo

El acceso a los contenidos en la nube resulta enriquecedor

Agradezco poder consultar la información de forma innovadora

Prefiero poder saltar de una información a otra (como al navegar por internet)

Este tipo de instalaciones en ningún caso me ayudan a mejorar mis competencias para acceder a la información digitalizada

21. Tras el visionado del docuclip, ¿con qué grado de precisión crees que puedes definir el concepto de «entorno inmersivon?
Nada preciso
Algo preciso
Preciso
Bastante preciso
Muy preciso

22. Tras el visionado del docuclip, ¿con qué grado de precisión crees que puedes definir el concepto de «instalación interactiva»?

Nada preciso

Algo preciso

Preciso

Bastante preciso

Muy preciso 


\section{BLOQUE IV. SUGERENCIAS SOBRE FUTURAS LÍNEAS DE ACTUACIÓN}

23. Responde en qué medida estás de acuerdo con las siguientes cuestiones en relación al posible potencial de las instalaciones interactivas en entornos inmersivos (1 [Nada/Casi nada] y 5 [Mucho])
1. Nada/
Casi nada
2. Poco
3. Algo
4. Bastante
5. Mucho

Me gustaría que este tipo de instalación también se utilizara para impartir docencia (es decir, la materia de estudio obligatorio en el colegio, instituto, universidad)

Este tipo de instalación solo debería ser accesible en los museos e instituciones culturales

Estas instalaciones mejorarán el futuro papel educativo de los museos

Los visitantes discapacitados también podrían disfrutar de estas instalaciones por su componente multisensorial y por la libertad de movimiento

El diseño de este tipo de instalaciones permitiría exhibir todo tipo de contenidos (no solo la enciclopedia del Prado)

Este tipo de instalación sería adecuada únicamente como actividad de ocio

Este tipo de experiencias inmersivas pueden adaptarse tanto a niños y jóvenes como a un público especializado

La presencia de estas instalaciones fomentaría las visitas a los museos

24. ¿En qué punto del recorrido expositivo preferirías acceder a la instalación EncyCloudPrado?

Al inicio de la visita Otra opción no contemplada en el anterior listado (especificar)
Cuando me encuentre hacia la mitad del recorrido expositivo

Al final de la exposición

25. ¿Tienes alguna sugerencia para mejorar EncyCloudPrado? 